like disasters. The LRSM design now supports engagement with lay responders very early in post-event period, and informed by continual findings.

Conclusion The LRSM provides a structured framework to capture information about witnessing a SCA from the lay-responders involved the role they played, actual clinical records, and to identify areas of support for lay-responder's residual mental health. It potentially goes beyond cardiac arrest situations and may prove helpful to psychological first aid providers and other public health organisations identifying and referring people to appropriate resources.

Conflict of interest None

Funding Employer - Peel Regional Paramedic Services.

\section{HEALTH ECONOMIC ANALYSIS OF LOW-DOSE METHOXYFLURANE (LDM) FOR THE TREATMENT OF MODERATE-TO-SEVERE TRAUMA PAIN IN SWEDEN}

${ }^{1} \mathrm{~A}$ Xia, ${ }^{1} \mathrm{D}$ Hooker, ${ }^{1} \mathrm{~S}$ Dickerson*, ${ }^{2} \mathrm{~L}$ Hegarty, ${ }^{2} \mathrm{D}$ Fielden, ${ }^{2} \mathrm{~L}$ Strait. ${ }^{1}$ Mundipharma International Ltd, UK; ${ }^{2}$ Covance Market Access Services Inc., UK

\subsection{6/10.1136/bmjopen-2018-EMS.28}

Aim Inadequately treated trauma pain can lead to prolonged and unnecessary patient suffering, as well as lengthen hospital stay. ${ }^{1,2}$ LDM (Penthrox $\left.{ }^{\circledR}\right)$ is an inhaled analgesic indicated for emergency relief of moderate-to-severe pain in conscious adult patients with trauma and associated pain. ${ }^{3}$ The aim of this study was to assess the impact of introducing LDM in the pre-hospital setting for a European healthcare system.

Method A health economic model was developed from the Swedish healthcare system perspective to evaluate outcomes and resource use associated with LDM vs standard of care (morphine injection, fentanyl nasal spray and ketamine injection) over a 5 year time horizon. Model inputs included time to first pain medication treatment, drug acquisition, administration and equipment costs, practitioner rates and adverse event costs. Inputs were derived from published data supplemented by the results of a retrospective chart review and physician survey.

Results Based on a national Swedish population of $\sim 10 \mathrm{M}$ individuals, the model estimated that $\sim 600000$ patients would be eligible for LDM treatment in Year 1. Introducing LDM at a $1 \%$ uptake rate in Year 1, increasing $1 \%$ annually, was estimated to result in the avoidance of $\sim 15,600$ hours of moderate-to-severe pain over 5 years, allowing for the treatment of an additional $\sim 43000$ patients for moderate-tosevere pain. A modest cumulative 5 year budget impact increase is estimated at SEK $1 \mathrm{M}$.

Conclusion Introducing LDM as a treatment for moderate-tosevere trauma pain in the pre-hospital setting may result in fewer hours of patient pain and more patients treated at a modest budget increase.

\section{REFERENCES}

1. Sinatra R. Pain Med 2010;11(12):1859-71.

2. Sokolof C, et al. BMJ Open 2014:4:e004288.

3. PENTHROX $3 \mathrm{~mL}$ inhalation vapour, liquid. PENTHROX $3 \mathrm{~mL}$ inhalation vapour, liquid 2017. Available from: https://www.medicines.org.uk/emc/medicine/31391 (accessed 19 April 2017)

Conflict of interest None

Funding Mundipharma International Ltd

\section{9 OUT-OF-HOSPITAL CARDIAC ARREST IN PATIENTS WITH PSYCHIATRIC DISORDER - CHARACTERISTICS AND OUTCOMES}

${ }^{1} \mathrm{CA}$ Barcella*${ }^{1}{ }^{\mathrm{GH}}$ Mohr, ${ }^{2,3} \mathrm{~K}$ Kragholm, ${ }^{1,4} \mathrm{P}$ Blanche, ${ }^{1,5} \mathrm{M}$ Wissenberg, ${ }^{3} \mathrm{SM}$ Hansen, ${ }^{1,5} \mathrm{~F}$ Folke, ${ }^{2,3,6} \mathrm{C}$ Torp-Pedersen, ${ }^{7} \mathrm{LV}$ Kessing, ${ }^{1,8} \mathrm{GH}$ Gislason, ${ }^{1} \mathrm{~KB}$ Sondergaard. ${ }^{1}$ Department of Cardiology, Copenhagen University Hospital Herlev and Gentofte, Denmark; ${ }^{2}$ Department of Cardiology, Aalborg University Hospital, Denmark; ${ }^{3}$ Unit for Epidemiology and Biostatics, Aalborg University Hospital, Aalborg, Denmark; ${ }^{4}$ Department of Biostatistics, University of Copenhagen, Denmark; ${ }^{5}$ Emergency Medical Services: The Capital Region of Denmark, Copenhagen, Denmark; ${ }^{6}$ Institute of Health, Science and Technology, Aalborg University, Denmark; ${ }^{7}$ Psychiatric Centre Copenhagen, Copenhagen University Hospital Rigshospitalet, Denmark; ${ }^{8}$ The Danish Heart Foundation, Copenhagen, Denmark

\subsection{6/10.1136/bmjopen-2018-EMS.29}

Aim Survival after out-of-hospital cardiac arrest (OHCA) has increased in several countries following improvements in prehospital OHCA-management. We investigated overall and temporal changes in characteristics and outcomes in OHCApatients with previous psychiatric disorder.

Method We identified adult patients with OHCA of presumed cardiac cause from the Danish Cardiac Arrest Registry from 2001 through 2014. Using multivariable logistic regression we calculated odds ratio (OR) for cardiopulmonary resuscitation (CPR), survival upon hospital arrival, 30 day and 1 year survival after OHCA in patients with and without a history of psychiatric disorder identified by discharge diagnosis up to 10 years before OHCA.

Results Of 28.955 OHCAs, 4037 (13.9\%) had a known psychiatric disorder. Psychiatric patients were younger, less likely to have bystander-witnessed OHCA $(44.1 \%$ vs $51 \%)$ and shockable heart rhythm $(14.8 \%$ vs $27.1 \%)$, but same degree of arrest in private home $(64.5 \%$ vs $64.8 \%)$ and estimated time interval to first-rhythm-analysis by EMS-crew (12 min) compared to non-psychiatric OHCA-patients. In fully-adjusted models, psychiatric patients had the same probability of receiving bystander CPR (OR 0.93 [95\% confidence interval, $0.83-$ $1.02]$ ), but lower probability of survival upon hospital arrival, 30 day and 1 year survival: OR 0.79 [0.71-0.89], 0.54 [CI: 0.46 to 0.64$]$ and 0.49 [0.41-0.59], respectively. Temporal trends showed that the gap in 30 day and 1 year survival between psychiatric and non-psychiatric OHCA-patients became evident after 2007.

Conclusion Survival following OHCA among psychiatric patients is considerably lower compared with non-psychiatric OHCA-patients and the gap seems to widen over time despite the recent efforts to improve OHCA-management.

Conflict of interest None

Funding None

\section{WHEN EVALUATING THE LEVEL OF LACTATE: COULD VENOUS BLOOD GAS BE EQUATED TO ARTERIAL BLOOD GAS?}

${ }^{1}$ Ninna HG Christensen*, ${ }^{1}$ Mads Lumholdt, ${ }^{2}$ Kjeld Asbjørn Damgaard. ${ }^{1}$ Department of Anaesthesiology, North Denmark Regional Hospital; ${ }^{2}$ Emergency department, North Denmark Regional Hospital

\subsection{6/10.1136/bmjopen-2018-EMS.30}

Aim Measurement of lactate level is an essential tool in the clinical assessment of patients. Lactate levels greater than $2 \mathrm{mmol} / \mathrm{L}$ represent hyperlactataemia whereas lactic acidosis is generally defined as a serum lactate concentration above 
$4 \mathrm{mmol} / \mathrm{L}$. The lactate level is most frequently measured in the arterial blood gas (ABG). Aim of this study is to evaluate the agreement of venous blood gas (VBG) lactate compared to ABG lactate.

Method Prospectively collected data from twenty patients were included in this study. All patients were admitted to the emergency department. ABG and three types of VBG samples were collected from each patient. To compare the VBG samples, processing was done in three different ways; VBG1 was held steady and analysed within $5 \mathrm{~min}, \mathrm{VBG} 2$ was tilted for $5 \mathrm{~min}$ and analysed within $7 \mathrm{~min}, \mathrm{VBG} 3$ was held steady and analysed after $15 \mathrm{~min}$. ABG and VBG samples were compared using Bland-Altman plot.

Results The Bland-Altman plot showed narrow 95\% limits of agreement and average differences in lactate of $-0,24,-0,25$ and $-0,33$ between $A B G$ and VBG1-3, respectively.

Conclusion Venous blood lactate is a valid parameter when measuring the level of lactate. However larger studies are needed to further evaluate the reliability regarding patients with severe hyperlactataemia.

Conflict of interest None declared

Funding This study did not receive external funding.

\section{OUT-OF-HOSPITAL TREATMENT OF ACUTE FOREIGN BODY AIRWAY OBSTRUCTION IN ADULTS: A STRUCTURED LITERATURE REVIEW}

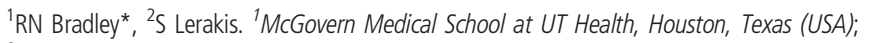
${ }^{2}$ Emory University, Atlanta, Georgia (USA)

\subsection{6/10.1136/bmjopen-2018-EMS.31}

Aim There were 4700 deaths due to choking in the United States in 2012. This study was a structured literature review with the objective of determining whether in adults, either conscious or unconscious, with acute foreign body airway obstructions, if any specific resuscitation technique, compared to other techniques, leads to different outcomes.

Method We developed an a priori search definition, and searched PubMed, Google, and OneSearch@IU. We sought additional articles by reviewing the reference lists of articles that we included. We included articles if they addressed original research of treatment of foreign body airway obstruction in adults. We excluded articles if the skill was not applicable to an out-of-hospital rescuer or if there was no abstract available in English. For those articles that met our criteria, two investigators independently collected the results and assessed the quality of the evidence.

Results We identified 534 articles for screening and performed full-text reviews on 64 . We included 44 articles in our qualitative synthesis. We found one fair quality study that supported the use of abdominal thrusts, two fair quality studies that supported back blows, and one fair quality study that supported chest thrusts.

Conclusion Rescuers attempting to resolve a complete foreign body airway obstruction in a conscious adult should provide back blows and either abdominal and/or chest thrusts to the victim. Rescuers attempting to resolve a complete foreign body airway obstruction in an unconscious adult should provide CPR and use a finger sweep if a foreign body is seen in the mouth.

Conflict of interest RNB and SL are both employed by their respective medical schools. Some travel expenses are reimbursed as SL is a member and RNB is the chair of the Resuscitation Subcouncil of the American Red Cross Scientific Advisory Council.

Funding None

\section{DERIVATION OF A NOVEL INPATIENT MORTALITY PREDICTION MODEL FOR EMERGENCY DEPARTMENT PATIENTS IN SINGAPORE}

${ }^{1}$ Stella Xinzi Wu*, ${ }^{1,2}$ Nan Liu, 1,3Marcus Eng Hock Ong. 'Duke-NUS Medical School, National University of Singapore, Singapore; ${ }^{2}$ Health Services Research Centre, Singapore Health Services, Singapore; ${ }^{3}$ Department of Emergency Medicine, Singapore General Hospital, Singapore

\subsection{6/10.1136/bmjopen-2018-EMS.32}

Aim Inpatient mortality is an indicator of hospital performance and patient care. In this retrospective cohort study, we aimed to develop and validate an inpatient mortality model for use during ED consultation to efficiently risk stratify patients for better care and resource allocation.

Method Data was extracted from the Electronic Health Records (unified patient care data) of Singapore General Hospital in the year of 2014. Patients admitted through the ED were included and patients $<21$ were excluded. Variables, such as demographics, comorbidities, socioeconomic status and laboratory tests, were selected through literature review and clinician judgement before analysis with univariable and multivariable logistic regression. The model was assessed with receiver operating characteristic area under the curve.

Results Among the 35699 patients admitted from ED in 2014, 690 died in-hospital. Univariate-analysis showed males, lower socioeconomic status, multiple comorbidities and increased acuity of illness as significant variables contributing to inpatient mortality. The final model included gender, use of medifund (financial aid), Charlson Comorbidity Index (CCI), albumin, creatinine, white blood cell counts, and number of ED visits within the past 1 year. This model (AUC 0.840) performed the best compared to other scores, including just using CCI, age, gender and principal diagnosis (AUC 0.723).

Conclusion A novel model for predicting inpatient mortality could effectively risk stratify patients early in the ED. This model may have future applications to improve management and disposition.

Conflict of interest None

Funding None

\section{INTERACTION EFFECTS BETWEEN TARGETED TEMPERATURE MANAGEMENT AND HYPERTENSION ON SURVIVAL OUTCOMES AFTER OUT-OF-HOSPITAL CARDIAC ARREST}

Jung Eujene*. Chonnam National University Hospital Clinical Fellow

\subsection{6/10.1136/bmjopen-2018-EMS.33}

Aim Targeted temperature management (TTM) currently represents the most efficacious treatment to improve neurological recovery in comatose patients who have return of spontaneous circulation (ROSC) after out-of-hospital cardiac arrest (OHCA). The effect of TTM with HTN have not been reported. This study aimed to investigate whether the effect of TTM on neurological recovery after OHCA differed between patients with or without HTN. 